\title{
Value of Left Atrial Appendage Function Measured by Transesophageal Echocardiography for Prediction of Atrial Fibrillation Recurrence after Radiofrequency Catheter Ablation
}

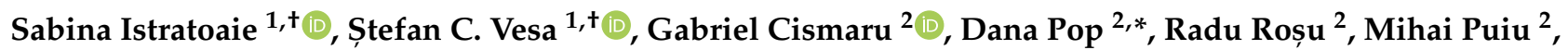 \\ Diana Pepine ${ }^{2}$, Cristina Ciobanu ${ }^{2}$, Ioan A. Minciuna ${ }^{2}$, Gelu Simu ${ }^{2}$, Dumitru Zdrenghea ${ }^{2}$ and
} Anca D. Buzoianu ${ }^{1}$ (D)

1 Department of Pharmacology, Toxicology and Clinical Pharmacology, Iuliu Haţieganu University of Medicine and Pharmacy, 400337 Cluj-Napoca, Romania; sabina.istratoaie@gmail.com (S.I.); stefanvesa@gmail.com (S.C.V.); abuzoianu@umfcluj.ro (A.D.B.)

2 5th Department of Internal Medicine, Cardiology-Rehabilitation, Iuliu Haţieganu University of Medicine and Pharmacy, 400066 Cluj-Napoca, Romania; gabi_cismaru@yahoo.com (G.C.); rosu.radu1053@gmail.com (R.R.); puiu.mihai@yahoo.com (M.P.); diana.pepine@yahoo.com (D.P.); cristina.cbn@yahoo.com (C.C.); iaminciuna@gmail.com (I.A.M.); simugelu@yahoo.com (G.S.); dzdrenghea@yahoo.com (D.Z.)

* Correspondence: pop67dana@gmail.com

+ These authors equally contributed to this work.

Citation: Istratoaie, S.; Vesa, Ș.C.; Cismaru, G.; Pop, D.; Roșu, R.; Puiu, M.; Pepine, D.; Ciobanu, C.;

Minciuna, I.A.; Simu, G.; et al. Value of Left Atrial Appendage Function

Measured by Transesophageal

Echocardiography for Prediction of Atrial Fibrillation Recurrence after Radiofrequency Catheter Ablation. Diagnostics 2021, 11, 1465. https:// doi.org/10.3390/diagnostics11081465

Academic Editor: Michael Henein

Received: 31 July 2021

Accepted: 11 August 2021

Published: 13 August 2021

Publisher's Note: MDPI stays neutral with regard to jurisdictional claims in published maps and institutional affiliations.

Copyright: (C) 2021 by the authors. Licensee MDPI, Basel, Switzerland. This article is an open access article distributed under the terms and conditions of the Creative Commons Attribution (CC BY) license (https:// creativecommons.org/licenses/by/ $4.0 /)$.
Abstract: Atrial fibrillation (AF) recurrence after radiofrequency catheter ablation (RFCA) remains a challenging issue. This study aims to explore the left atrial appendage function by transesophageal echocardiography (TEE) and assess its value in predicting AF recurrence following RFCA in paroxysmal AF patients. Eighty-one patients with paroxysmal AF that underwent RFCA were recruited. TEE was performed before ablation with the assessment of left atrial appendage emptying flow velocity (LAAeV). AF recurrence occurred in 24 patients (29.6\%) within 12 months after RFCA. The left atrium diameter (LAD) and left atrium volume index (LAVI) were both significantly higher in the recurrence group compared to the non-recurrence group, while the LAAeV was significantly lower in the recurrence group. LAD, LAVi and LAAeV were univariately significant risk factors for AF recurrence after ablation. Based on receiver operating curve (ROC), $\mathrm{LAAeV}<40.5 \mathrm{~cm} / \mathrm{s}$, $\mathrm{LAVi}>40.5 \mathrm{~mL}$ and LAD $>41 \mathrm{~mm}$ were identified as cut-off values for predicting AF recurrence. In multivariate regression analysis LAAeV $<40.5 \mathrm{~cm} / \mathrm{s}$ (HR 8.194, 95\% CI 2.980-22.530, $p<0.001$ ) was identified as the only statistically significant independent predictor of AF recurrence, as the statistical significance threshold was not achieved for LAVI $>40.5 \mathrm{~mL}$ and LAD $>41 \mathrm{~mm}(p=0.319 ; p=0.507$, respectively). A low LAAeV was the only important independent predictor of AF recurrence within 1 year after first RFCA.

Keywords: atrial fibrillation; transesophageal echocardiography; left atrial appendage emptying flow velocity

\section{Introduction}

Atrial fibrillation (AF) is the most common sustained cardiac arrhythmia, with a lifetime AF risk of about one in three individuals of European ancestry over 55 years of age [1,2]. The presence of AF is associated with impaired quality of life and increased risk of stroke, heart failure and cardiovascular mortality. RFCA targeting pulmonary vein isolation is a well established effective therapeutic option for paroxysmal AF. However, the success rate of PVI ranges between 50 and 80\% [3,4]. Therefore, evaluation of predictive factors of AF recurrence is important to optimize the selection of appropriate patients as well as to improve the success rate of RFCA. It has been reported that left atrial dilation and decreased left atrium (LA) function is correlated with a high AF recurrence rate [5-7]. In addition to left atrial appendage (LAA) playing important role in predicting cardioembolic 
stroke, LAA can also reflect LA function and severity of the LA remodeling in patients with AF $[8,9]$. However, information on parameters to assess the role of LAA function in AF recurrence after RFCA is limited. The imaging technique of first choice to evaluate LAA structure and function is transesophageal echocardiography. The function of the LAA is most commonly determined by measuring emptying velocity with pulsed-wave Doppler. The purpose of this study was to assess whether left atrial appendage emptying flow velocity (LAAeV) may provide predictive value for paroxysmal AF recurrence within 1 year after first RFCA.

\section{Materials and Methods}

\subsection{Patient Selection}

Patients with symptomatic, paroxysmal AF who had undergone the first RFCA for AF in the Cardiology Department of the Rehabilitation Hospital from Cluj-Napoca, between December 2018 and January 2020 were enrolled. AF was defined as paroxysmal if AF terminated within 7 days of onset (spontaneously or with intervention). The exclusion criteria were as follows: poor quality of TEE image acquisition, reversible causes for $\mathrm{AF}$, prior cardiac surgery, severe valvular disease, congenital heart disease and decompensated heart failure. The study design was approved by the Ethics Committee of Rehabilitation Hospital Cluj-Napoca and written informed consent prior to the procedure and participation in the study was obtained from each patient.

\subsection{Transthoracic and Transesophageal Echocardiogram}

All patients underwent both transthoracic echocardiography (TTE) and transesophageal echocardiography (TEE) within $24 \mathrm{~h}$ before RFCA. All the parameters were performed according to the American Society of Echocardiography guidelines. During TTE left ventricular end-systolic, and end-diastolic volumes (LVESV and LVEDV, respectively), and the left ventricular ejection fraction (LVEF) were measured. Left atrial diameter (LAD) was obtained on the parasternal long-axis view, while the left atrial volume (LAV) was calculated by the modified Simpson's method. LAVI was obtained using body surface area correction. During TEE evaluation, LAA was visualized from the mid-oesophageal view at multiple omni-plane angles, between 45 and $100^{\circ}$. For pulsed-wave Doppler interrogation, the angle that provided the longest LAA dimension was used. The LAAeV was defined as a late diastolic positive outflow signal, and it was measured using a $4 \mathrm{~mm}$ sample volume positioned at the entry of the LAA orifice. In patients with paroxysmal AF at the time of TEE, LAAeV was described as the average value of five consecutive cardiac cycles (Figure 1). All cardiac ultrasound examinations were performed on a Philips Affiniti 50 (Philips Healthcare, Best, The Netherlands) with a 2-4 MHz microconvex transducer for ETT and a $7 \mathrm{MHz}$ transducer for TEE. All echocardiographic measurements were conducted by the same two echocardiogram experts. 


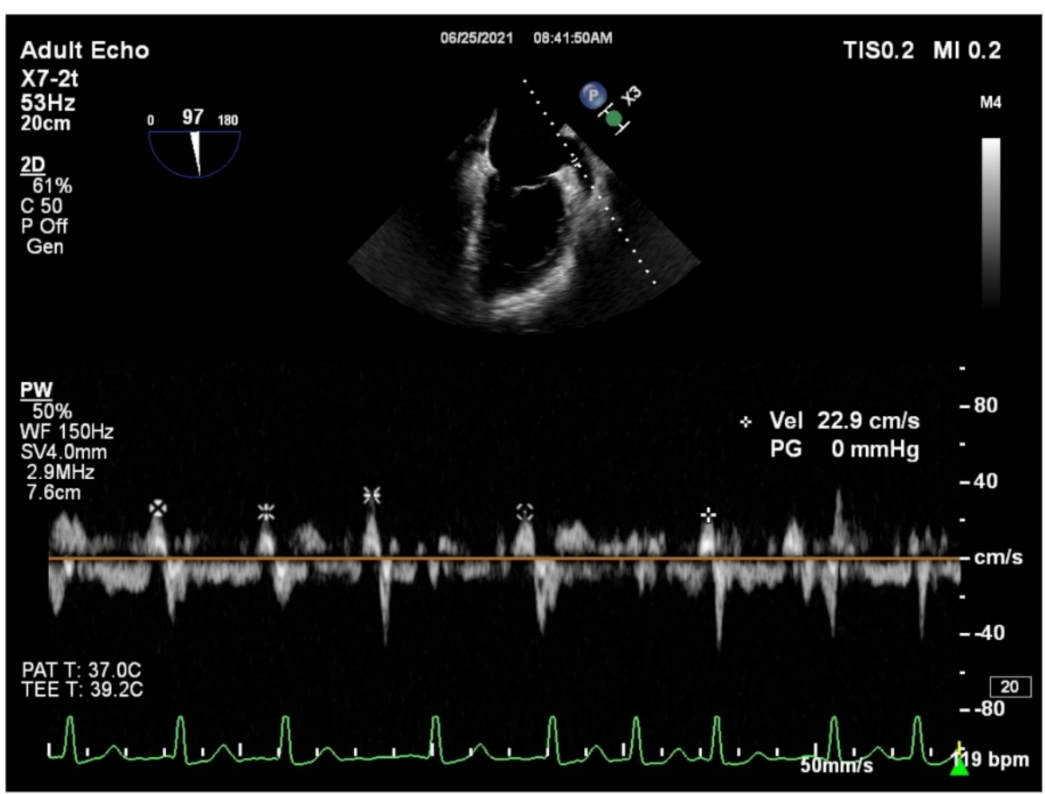

Figure 1. Example of measuring the left atrial appendage emptying velocity (LAAeV). The LAAeV was measured by pulsed-wave Doppler interrogation at the entry of the left atrial appendage at $84^{\circ}$ $(22.9 \mathrm{~cm} / \mathrm{s})$.

\subsection{RFCA}

Three-dimensional mapping of LA was performed with an Ensite NAVX Velocity (Saint-Jude Medical, Saint Paul, MN, USA) or a CARTO 3 (Biosense Webster, Diamond Bar, CA, USA) system. To create ablation lesions an open-irrigated 7-french $3.5 \mathrm{~mm}$ ablation catheter (Navistar Thermocol and Thermocool Smarttouch, Biosense Webster, Diamond Bar, CA, USA) or the FlexAbility irrigated ablation catheter (Saint-Jude Medical, Saint Paul, MN, USA) were used. Continuous radiofrequency ablation was performed by encircling the ipsilateral PVs until electrical isolation was achieved. The isolation of the electric field was considered to be complete in the absence of PV potential within each antrum, using a circular mapping catheter (LassoNav or PentaRay, Biosense Webster, Diamond Bar, CA, USA).

\subsection{Follow-Up}

All patients were scheduled for clinical visits to undergo 12-lead electrocardiography (ECG) and 24-h Holter recording at 3, 6, and 12 months after RFCA. Patients were also advised to present to our cardiology department if they experienced palpitations. AF recurrence was defined as any atrial tachyarrhythmia that lasted for more than $30 \mathrm{~s}$ captured by ECG or Holter monitoring. Transient episodes of arrhythmia recurrence during the first 3 months after ablation were counted as a blanking period.

\subsection{Statistical Analysis}

SPSS 21.0 statistical software (IBM, Chicago, IL, USA) was used to analyze the data. Continuous variables were checked for normality of distribution (Shapiro-Wilk test, kurtosis and skewness coefficients) and are presented as mean \pm standard deviation, or median with interquartile range ( $25 \%, 75 \%$ percentiles). Categorical variables are described as frequency and percentage. Data were compared between the AF recurrence and non-recurrence groups using Mann-Whitney, Student's t test or chi-square tests, when appropriate. Univariate and multivariate Cox proportional risk regression models were used to identify the risk factors associated with AF recurrence. ROC (receiver operating curve) analysis was used to calculate the best cut-off value of predictors of AF recurrence. Inter-observer reliability analysis was carried out using Cronbach's alpha. A $p$ value of $<0.05$ was considered statistically significant. 


\section{Results}

A total of 81 consecutive patients with paroxysmal AF who underwent RFCA for the first time were enrolled, aged $55.3 \pm 9$ years, $48(59.3 \%)$ men and $33(40.7 \%)$ women. The median follow-up time was $12(11 ; 14)$ months. After the blanking period of 3 months, 24 patients $(29.6 \%)$ had had AF recurrence (recurrence group) and 57 patients $(70.4 \%)$ had maintained sinus rhythm (nonrecurrence group).

The clinical characteristics of patients with and without AF recurrence were compared in Table 1. There were no statistically significant differences in age, sex, body mass index (BMI), diabetes mellitus, hypertension, coronary heart disease, stroke, transient ischemic attack, duration of AF and medication between the two groups.

Table 1. Clinical characteristics after RFCA of AF.

\begin{tabular}{|c|c|c|c|c|}
\hline \multicolumn{2}{|c|}{ Baseline Characteristics } & $\begin{array}{c}\text { Recurrence } \\
\quad N=24\end{array}$ & $\begin{array}{c}\text { Non-Recurrence } \\
\quad \mathrm{N}=57\end{array}$ & $p$ \\
\hline \multicolumn{2}{|c|}{ Age (years) } & $55.9 \pm 9.8$ & $54.6 \pm 8.4$ & 0.5 \\
\hline \multicolumn{2}{|c|}{ Male, $n(\%)$} & $12(50)$ & $31(54.4)$ & 0.37 \\
\hline \multicolumn{2}{|c|}{ BMI $\left(\mathrm{kg} / \mathrm{m}^{2}\right)$} & $26.6 \pm 2.5$ & $25.9 \pm 2.8$ & 0.27 \\
\hline \multicolumn{2}{|c|}{ Coronary artery disease } & $5(20.8)$ & $4(7)$ & 0.07 \\
\hline \multicolumn{2}{|c|}{ Hypertension, $n(\%)$} & $11(45)$ & $22(38.6)$ & 0.5 \\
\hline \multicolumn{2}{|c|}{ Diabetes mellitus, $n(\%)$} & $3(12.5)$ & $4(7)$ & 0.42 \\
\hline \multicolumn{2}{|c|}{ Stroke or TIA, $n(\%)$} & $3(12.5)$ & $2(3.5)$ & 0.12 \\
\hline \multicolumn{2}{|c|}{ Hyperlipidaemia, $n(\%)$} & $7(29.2)$ & $17(29.8)$ & 0.49 \\
\hline \multicolumn{2}{|c|}{ AF history (months) } & $34(10,55)$ & $36(11,45)$ & 0.38 \\
\hline \multirow{3}{*}{$\begin{array}{l}\text { Antiarrhythmic } \\
\text { Drug }\end{array}$} & Propafenone, $n(\%)$ & $5(20.8)$ & $12(21.1)$ & \multirow{3}{*}{0.127} \\
\hline & Amiodarone, $n(\%)$ & $5(20.8)$ & $22(38.6)$ & \\
\hline & Flecainide, $n(\%)$ & $11(45.8)$ & $12(21.1)$ & \\
\hline \multicolumn{2}{|c|}{ Betablocker, $n(\%)$} & $12(50)$ & $20(35.1)$ & 0.315 \\
\hline \multicolumn{2}{|c|}{ RAS inhibitors, $n(\%)$} & $11(45.8)$ & $23(40.4)$ & 0.834 \\
\hline \multicolumn{2}{|c|}{ Statin, $n(\%)$} & $11(45.8)$ & $25(45.6)$ & 1 \\
\hline
\end{tabular}

The LAD and LAVI were significantly greater in the recurrence than in the nonrecurrence group (Table 2$)$. The LAAeV was significantly $(p<0.05)$ lower for the patients with AF recurrence when compared to the patients who maintained a sinus rhythm (Table 2). However, no significant differences existed in the LVEDV, LVESV, and LVEF between the two groups. The Cronbach's alpha for LAD and LAVI were 0.895 and 0.861 , which shows good reliability. For LAAeV we calculated a Cronbach's alpha of 0.904 , which shows excellent reliability.

Table 2. Ultrasound parameters.

\begin{tabular}{cccc}
\hline Ultrasonic Parameters & $\begin{array}{c}\text { Recurrence } \\
\mathbf{N = \mathbf { 4 }}\end{array}$ & $\begin{array}{c}\text { Non-Recurrence } \\
\mathbf{N}=\mathbf{5 7}\end{array}$ & $\boldsymbol{p}$ \\
\hline LAD $(\mathrm{mm})$ & $39.8 \pm 5.7$ & $37.1 \pm 4.6$ & 0.03 \\
LAVI $\left(\mathrm{mL} / \mathrm{m}^{2}\right)$ & $43(37 ; 51)$ & $36(32 ; 40)$ & $<0.001$ \\
LVEDV $(\mathrm{mL})$ & $92(82 ; 101.5)$ & $94.5(83.5 ; 110.75)$ & 0.16 \\
LVESV $(\mathrm{mL})$ & $33(27.5 ; 43.5)$ & $35(29.5 ; 58.5)$ & 0.13 \\
LVEF $(\%)$ & $61(55 ; 64.7)$ & $60(55 ; 63)$ & 0.497 \\
LAAeV $(\mathrm{cm} / \mathrm{s})$ & $37.9 \pm 8.5$ & $49.4 \pm 6.8$ & 0.001 \\
\hline
\end{tabular}


To investigate the associations between the various parameters and AF recurrence, a Cox proportional hazards regression analysis was performed. In the univariate analysis, high LAD and LAVI, as well as low LAAeV, were significant risk factors for AF recurrence (Table 3).

Table 3. Univariate analysis for AF recurrence.

\begin{tabular}{ccc}
\hline & HR (95\% CI) & $p$ \\
\hline Age & $1.021(0.974-1.072)$ & 0.386 \\
Gender & $1.565(0.703-3.484)$ & 0.273 \\
Coronary artery disease & $2.626(0.979-7.042)$ & 0.055 \\
Hypertension & $1.263(0.566-2.820)$ & 0.568 \\
Diabetes mellitus & $1.655(0.493-5.557)$ & 0.415 \\
Stroke & $3.944(0.872-17.833)$ & 0.075 \\
Hyperlipidaemia & $1.238(0.449-3.415)$ & 0.680 \\
Propafenone & $1.346(0.322-5.634)$ & 0.684 \\
Flecainide & $0.787(0.188-3.295)$ & 0.743 \\
Amiodarone & $2.290(0.638-8.212)$ & 0.204 \\
Betablocker & $1.638(0.736-3.647)$ & 0.227 \\
RAS inhibitors & $1.209(0.541-2.698)$ & 0.644 \\
Statin & $1.015(0.455-2.266)$ & 0.971 \\
LAD & $1.112(1.025-1.207)$ & 0.011 \\
LAVi & $1.126(1.072-1.183)$ & $<0.001$ \\
LVEF & $1.031(0.948-1.122)$ & 0.475 \\
LVEDV & $1.022(0.993-1.051)$ & 0.142 \\
LVESV & $1.027(0.998-1.057)$ & 0.068 \\
LAAeV & $0.856(0.807-0.908)$ & $<0.001$ \\
\hline
\end{tabular}

The variance inflation factors of LAD, LAVI, LAAeV were calculated and, because these factors were less than 5, no multiple collinearities existed (Table 4).

Table 4. Collinearity analysis.

\begin{tabular}{cccc}
\hline & LAD & LAVI & LAAeV \\
\hline VIF & 1.842 & 1.693 & 1.671 \\
\hline
\end{tabular}

The variance inflation factors of LAD, LAVI, LAAeV were calculated and, because these factors were less than 5, no multiple collinearities existed (Table 4).

ROC curve analysis was used to evaluate the predictive efficiency of LAVI, LAAeV and LAD. The best cut-off value of the LAVI for the prediction of an AF recurrence was $40.5 \mathrm{~mL} / \mathrm{m}^{2}$ with a sensitivity of $63 \%$, specificity of $77 \%$ (AUC 0.776 ). An LAAeV cutoff value of $40.5 \mathrm{~cm} / \mathrm{s}$ was selected for predicting rhythm outcome after catheter ablation with $89 \%$ sensitivity and $75 \%$ specificity (AUC 0.869 ). A LAD cutoff value over $41 \mathrm{~mm}$ was selected for predicting rhythm outcome after catheter ablation with $50 \%$ sensitivity and $84 \%$ specificity (AUC 0.670 ). The AUC for LAD was statistically significantly lower than the AUC for LAAeV $(p=0.001)$, but not than the AUC for LAVI $(p=0.146)$. The AUC for LAVI was not statistically significantly lower than the AUC for LAAeV $(p=0.184)$.

LAVI, LAAeV and LAD were introduced in multivariate analysis, after they were redefined according to their cut-off values (Table 5). LAAeV under $40.5 \mathrm{~cm} / \mathrm{s}$ was the only independent predictor for recurrence after radiofrequency ablation of AF. 
Table 5. Multivariate analysis for AF recurrence.

\begin{tabular}{cccccc}
\hline & \multirow{2}{*}{ B } & \multirow{2}{*}{ HR } & \multicolumn{2}{c}{$\mathbf{9 5 . 0 \%}$ CI for HR } \\
& & & & Min & Max \\
\hline Age & -0.025 & 0.375 & 0.976 & 0.924 & 1.030 \\
Gender (female) & 0.852 & 0.071 & 2.345 & 0.930 & 5.913 \\
LAD $>41 \mathrm{~mm}$ & 0.731 & 0.165 & 2.077 & 0.741 & 5.820 \\
LAVI $>40.5 \mathrm{~mL} / \mathrm{m}^{2}$ & 0.358 & 0.467 & 1.430 & 0.545 & 3.755 \\
LAAeV $<40.5 \mathrm{~cm} / \mathrm{s}$ & 2.150 & $<0.001$ & 8.588 & 3.125 & 23.599 \\
\hline Abbreviations: $\mathrm{B}$, unstandardized regression coefficient; $\mathrm{P}$, coefficient of statistical significance; HR, hazard ratio
\end{tabular}

Abbreviations: B, unstandardized regression coefficient; $\mathrm{P}$, coefficient of statistical significance; HR, hazard ratio $\mathrm{CI}$, confidence interval.

\section{Discussion}

Our study demonstrated that (1) LAD and LAVI were significantly elevated in patients with AF recurrence compared with those without recurrence, whereas LAAeV was significantly decreased in the recurrence than non-recurrence group; (2) the cut off points with the highest predictive efficacy for AF recurrence were $\mathrm{LAAeV}<40.5 \mathrm{~cm} / \mathrm{s}$, $\mathrm{LAVi}<40.5 \mathrm{~mL} / \mathrm{m}^{2}$ and LAD $>41 \mathrm{~mm}$; (3) only LAAeV was identified as independent predictor of AF recurrence after RFCA.

Even though RFCA is an effective therapeutic approach for patients with paroxysmal $\mathrm{AF}$, recurrence of $\mathrm{AF}$ still remains problematic. In this study, the rate of $\mathrm{AF}$ recurrence following RFCA was $29.6 \%$, which is consistent with previous research [10]. The recurrence rate after catheter ablation is higher for patients with persistent $\mathrm{AF}$, and thus more risk factors have been established for AF recurrence [11,12]. We included only patients with paroxysmal $\mathrm{AF}$ in order to explore novel predictors of $\mathrm{AF}$ recurrence.

It is well known that LA enlargement is associated with AF recurrence after ablation. A meta-analysis of 22 studies has reported left atrium diameter to be a predictor of AF recurrence after ablation [5]. However, as LA dilation is asymmetric, the antero-posterior diameter is thought to underestimate LA size, whereas LA volume is more accurate in reflecting the size of the LA. Prior evidence showed that LAVI rather than LAD was related to AF recurrence following RFCA $[13,14]$. Although TTE can provide useful information for the detection of patients at risk for AF recurrence, LA volume is still determined using geometrical assumption, thus precisely estimating the LA structural dilation is challenging. Moreover, recent evidence shows that LA dysfunction is a more sensitive predictor for AF recurrence than LA size. Meanwhile, it is widely accepted that LAAeV measured by TEE can reflect not only LAA function but also LA function and severity of the LA remodeling. The LAA also presents contractile properties and a greater distensibility than the LA, that contributes to modulating LA pressure [15].

Kanda et al. used LAAeV as a surrogate factor of the LA function and were the first that demonstrated that a low LAAeV is associated with AF recurrence after catheter ablation [16]. They determined that the best cut-off value of the LAAeV for the prediction of AF recurrence was $28 \mathrm{~cm} / \mathrm{s}$ (sensitivity $62 \%$, specificity $69 \%$ ). However, they included patients with persistent $\mathrm{AF}$, probably with more severe LA remodeling compared to patients with paroxysmal AF, thus the LAAeV cut-off value they obtained is lower than in our study. Similar to our study were the results reported by He et al. that that also enrolled patients with paroxysmal AF and found that the best $\mathrm{LAAeV}$ cut off value in predicting AF recurrence was $39.2 \mathrm{~cm} / \mathrm{s}$ (se 75, Sp 82) [17]. However, they showed that not only LAAeV but also LAVi and the left atrial appendage ejection fraction are significant factors to predict $\mathrm{AF}$ recurrence following radiofrequency ablation.

Xin-Xin et al. evaluated whether LAAeV and/or NT-proBNP levels are associated with AF recurrence after RFCA [18]. They showed that for patients with persistent AF, a low LAAeV combined with elevated plasma NT-proBNP concentrations can predict rhythm outcome following catheter ablation, while for patients with paroxysmal $\mathrm{AF}$, a low LAAeV was the only independent predictor of CA efficacy. 
Another study that included 193 patients who underwent successful electrical cardioversion found a similar cutoff for LAAeV of $>40 \mathrm{~cm} / \mathrm{s}$ measured by TEE that predicted long-term SR [19].

Patients with paroxysmal AF are usually in the early stages of LA remodeling. Chronic pressure overload causes LA enlargement, while impairment of LA function precedes LA dilatation. Therefore, functional parameters, such as LAAeV, may be more sensitive markers for AF recurrence after ablation than LA size, particularly in patients with paroxysmal AF. Our findings support this hypothesis, since LAVI and LAD were not associated with rhythm recurrence based on multivariate logistic regression and only LAAeV independently predicted AF recurrence.

Although the value of LAA in AF recurrence following radiofrequency ablation is of major importance, it has been scarcely explored. In practice, preprocedural observation of LAA by TEE is conventionally performed to detect thrombus formation. An LAAeV of less than $40 \mathrm{~cm} / \mathrm{s}$ best predicts thromboembolic risk, while a value of more than $40 \mathrm{~cm} / \mathrm{s}$ is correlated with successful AF cardioversion and sinus rhythm maintenance [20-22]. However, there is no clear cutoff that would contraindicate the ablation procedure. The ablation is only contraindicated if an LAA thrombus is found because the procedure poses a risk of thromboembolic stroke due to thrombus mobilization caused by catheter manipulation. If the LAAeV is less than $40 \mathrm{~cm} / \mathrm{s}$ and additionally spontaneous contrast is present in the LAA, a CT or intracardiac echocardiogram should be performed before ablation to rule out an LAA thrombus. Therefore, when LAAeV is less than the cut-off value of 40.5 , it should not only raise the concern of a thrombus formation but also it might identify patients at risk to develop AF recurrence after RFCA. Moreover, if further studies on larger cohorts find a similar high AF recurrence rate based on low LAAeV, physicians may consider not performing RFCA. Identifying new risk predictors of AF recurrence is critical as it may contribute to a better selection of patients to undergo RFCA and to improve the success rate of catheter ablation.

The current study presented several limitations. Although we closely monitored patients using periodic ECG and Holter monitoring, asymptomatic episodes of AF may have been missed, which may have resulted in underestimation of the AF recurrence rate. We included a small cohort of patients, and thus our preliminary findings should be validated by future research including a larger number of patients.

\section{Conclusions}

In summary the left atrial diameter and the left atrial volume were significantly increased, while the left atrial appendage emptying flow velocity was significantly decreased in recurrence than in non-recurrence after radiofrequency ablation. A low left atrial appendage emptying flow velocity was the only independent predictor of AF recurrence within 1 year after radiofrequency ablation.

Author Contributions: Conceptualization, S.I. and D.P. (Dana Pop); methodology, G.C., R.R., M.P.; software, D.P. (Diana Pepine), C.C., I.A.M.; validation, G.S., D.P. (Dana Pop), D.Z. and A.D.B.; formal analysis, S.I., S.C.V.; investigation, S.I., G.C. and D.P. (Dana Pop); resources, R.R., M.P., I.A.M.; data curation, S.C.V.; writing—original draft preparation, S.I.; writing-review and editing, G.C., D.P. (Diana Pepine), D.P. (Dana Pop); visualization, G.S., C.C.; supervision, D.P. (Dana Pop), D.Z. and A.D.B. All authors have read and agreed to the published version of the manuscript.

Funding: This research received no external funding.

Institutional Review Board Statement: The study was conducted according to the guidelines of the Declaration of Helsinki and approved by the Ethics Committee of "Iuliu Haţieganu" University of Medicine and Pharmacy, Cluj-Napoca (138; 6 May 2019). 
Informed Consent Statement: Informed consent was obtained from all subjects involved in the study.

Acknowledgments: This paper was published under the frame of European Social Found, Human Capital Operational Programme 2014-2020, project no. POCU/380/6/13/125171.

Conflicts of Interest: The authors declare no conflict of interest.

\section{References}

1. Hindricks, G.; Potpara, T.; Dagres, N.; Arbelo, E.; Jeroen, J.B.; Carina, B.-L.; Giuseppe, B.; Manuel, C.; Gheorghe-Andrei, D.; Polychronis, E.D.; et al. 2020 ESC Guidelines for the diagnosis and management of atrial fibrillation developed in collaboration with the European Association for Cardio-Thoracic Surgery (EACTS). Eur. Heart J. 2021, 42, 373-498. [CrossRef] [PubMed]

2. Staerk, L.; Wang, B.; Preis, S.R.; Larson, M.G.; Lubitz, S.A.; Ellinor, P.T.; McManus, D.D.; Ko, D.; Weng, L.-C.; Lunetta, K.; et al. Lifetime risk of atrial fibrillation according to optimal, borderline, or elevated levels of risk factors: Cohort study based on longitudinal data from the Framingham Heart Study. BMJ 2018, 361, k1453. [CrossRef]

3. Calkins, H.; Kuck, K.H.; Cappato, R.; Brugada, J.; Camm, A.J.; Chen, S.-A.; Crijns, H.J.G.; Damiano, J.R.J.; Davies, D.W.; DiMarco, J.P.; et al. 2012 HRS/EHRA/ECAS Expert Consensus Statement on Catheter and Surgical Ablation of Atrial Fibrillation: Recommendations for Patient Selection, Procedural Techniques, Patient Management and Follow-up, Definitions, Endpoints, and Research Trial Design. Europace 2012, 14, 528-606. [CrossRef]

4. Ganesan, A.; Shipp, N.J.; Brooks, A.G.; Kuklik, P.; Lau, D.H.; Lim, H.S.; Sullivan, T.; Roberts-Thomson, K.C.; Sanders, P. Long-term Outcomes of Catheter Ablation of Atrial Fibrillation: A Systematic Review and Meta-analysis. J. Am. Heart Assoc. 2013, 2, e004549. [CrossRef]

5. Zhuang, J.; Wang, Y.; Tang, K.; Li, X.; Peng, W.; Liang, C.; Xu, Y. Association between left atrial size and atrial fibrillation recurrence after single circumferential pulmonary vein isolation: A systematic review and meta-analysis of observational studies. Europace 2012, 14, 638-645. [CrossRef]

6. Hoit, B.D. Left atrial size and function: Role in prognosis. J. Am. Coll. Cardiol. 2014, 63, 493-505. [CrossRef]

7. Kim, M.-N.; Lee, J.J.; Kim, S.-A.; Kim, Y.H.; Choi, J.I.; Park, S.-M.; Park, S.W.; Kim, Y.-H.; Shim, W.-J. The Difference of Predictors for Recurrence After Catheter Ablation of Non-Paroxysmal Atrial Fibrillation According to Follow-Up Period. Int. Heart J. 2014, 55, 312-318. [CrossRef]

8. Agmon, Y.; Khandheria, B.K.; Meissner, I.; Petterson, T.M.; O'Fallon, W.M.; Wiebers, D.O.; Seward, J.B. Are left atrial appendage flow velocities adequate surrogates of global left atrial function? A population-based transthoracic and transesophageal echocardiographic study. J. Am. Soc. Echocardiogr. 2002, 15, 433-440. [CrossRef]

9. Donal, E.; Grimm, R.A.; Yamada, H.; Yong, J.; Nassir, M.; Andrea, N.; James, D.T. Usefulness of Doppler assessment of pulmonary vein and left atrial appendage flow following pulmonary vein isolation of chronic atrial fibrillation in predicting recovery of left atrial function. Am. J. Cardiol. 2005, 95, 941-947. [CrossRef]

10. Arbelo, E.; Brugada, J.; Hindricks, G.; Maggioni, A.P.; Tavazzi, L.; Vardas, P.; Laroche, C.; Anselme, F.; Inama, G.; Jais, P.; et al. The Atrial Fibrillation Ablation Pilot Study: An European Survey on Methodology and results of catheter ablation for atrial fibrillation conducted by the European Heart Rhythm Association. Eur. Heart J. 2014, 35, 1466-1478. [CrossRef]

11. Vizzardi, E.; Curnis, A.; Latini, M.G.; Maria, G.; Salghetti, F.; Rocco, E.; Lupi, L.; Rovetta, R.; Quinzani, F.; Bonadei, I.; et al. Risk factors for atrial fibrillation recurrence: A literature review. J. Cardiovasc. Med. 2014, 15, 235-253. [CrossRef]

12. Brooks, A.G.; Stiles, M.K.; Laborderie, J.; Lau, D.H.; Kuklik, P.; Shipp, N.J.; Hsu, L.-F.; Sanders, P. Outcomes of long-standing persistent atrial fibrillation ablation: A systematic review. Heart Rhythm. 2010, 7, 835-846. [CrossRef]

13. Njoku, A.; Kannabhiran, M.; Arora, R.; Pratap, R.; Rakesh, G.; Dhanunjaya, L.; Paari, D. Left atrial volume predicts atrial fibrillation recurrence after radiofrequency ablation: A meta-analysis. Europace 2018, 20, 33-42. [CrossRef]

14. Istratoaie, S.; Roșu, R.; Cismaru, G.; Vesa, S.C.; Puiu, M.; Zdrenghea, D.; Pop, D.; Buzoianu, A.D. The Impact of Pulmonary Vein Anatomy on the Outcomes of Catheter Ablation for Atrial Fibrillation. Medicina 2019, 55, 727. [CrossRef] [PubMed]

15. Delgado, V.; Biase, L.D.; Leung, M.; Jorge, R.; Laurens, F.T.; Barbara, C.; Nassir, M.; Jeroen, J.B. Structure and Function of the Left Atrium and Left Atrial Appendage: AF and Stroke Implications. J. Am. Coll. Cardiol. 2017, 70, 3157-3172. [CrossRef] [PubMed]

16. Kanda, T.; Masuda, M.; Sunaga, A.; Masashi, F.; Osamu, I.; Shin, O.; Takayuki, I.; Tetsuya, W.; Mitsuyoshi, T.; Yasushi, S.; et al. Low left atrial appendage flow velocity predicts recurrence of atrial fibrillation after catheter ablation of persistent atrial fibrillation. $J$. Cardiol. 2015, 66, 377-381. [CrossRef]

17. He, Y.; Zhang, B.; Zhu, F.; Hu, Z.; Zhong, J.; Zhu, W. Transesophageal echocardiography measures left atrial appendage volume and function and predicts recurrence of paroxysmal atrial fibrillation after radiofrequency catheter ablation. Echocardiography 2018, 35, 985-990. [CrossRef] [PubMed]

18. Ma, X.-X.; Zhang, Y.-L.; Hu, B.; Jiang, W.-J.; Wang, M.; Zheng, D.-Y.; Zhu, M.-R.; Xue, X.-P. Association between left atrial appendage emptying velocity, N-terminal plasma brain natriuretic peptide levels, and recurrence of atrial fibrillation after catheter ablation. J. Interv. Card. Electrophysiol. 2016, 48, 343-350. [CrossRef]

19. Antonielli, E.; Pizzuti, A.; Pálinkás, A.; Mattia, T.; Gruber, N.; Claudio, M.; Albert, V.; Alessandro, B.; Nicola, G.; Halmai, L.; et al. Clinical value of left atrial appendage flow for prediction of long-term sinus rhythm maintenance in patients with nonvalvular atrial fibrillation. J. Am. Coll. Cardiol. 2002, 39, 1443-1449. [CrossRef] 
20. Fatkin, D.; Kelly, R.P.; Feneley, M.P. Relations between left atrial appendage blood flow velocity, spontaneous echocardiographic contrast and thromboembolic risk in vivo. J. Am. Coll. Cardiol. 1994, 23, 961-969. [CrossRef]

21. Mügge, A.; Kühn, H.; Nikutta, P.; Jochen, G.; Antonio, G.J.L.; Werner, G.D. Assessment of left atrial appendage function by biplane transesophageal echocardiography in patients with nonrheumatic atrial fibrillation: Identification of a subgroup of patients at increased embolic risk. J. Am. Coll. Cardiol. 1994, 23, 599-607. [CrossRef]

22. Chan, K.L. Transesophageal echocardiographic correlates of thromboembolism in high-risk patients with nonvalvular atrial fibrillation. The Stroke Prevention in Atrial Fibrillation Investigators Committee on Echocardiography. Ann. Intern. Med. 1998, $128,639-647$. 